\title{
CONCAVE FUNCTIONS OF PARTITIONED MATRICES WITH NUMERICAL RANGES IN A SECTOR
}

\author{
LEI Hou AND DENGPENG ZHANG
}

Abstract. We prove two inequalities for concave functions and partitioned matrices whose numerical ranges in a sector. These complement some results of Zhang in [Linear Multilinear Algebra 63 (2015) 2511-2517].

Mathematics subject classification (2010): 47A63, 15A45. trix.

Keywords and phrases: Rotfel'd theorem, concave function, numerical range, accretive-dissipative ma-

\section{REFERENCES}

[1] J. S. AUJla AND J. C. Bourin, Eigenvalues inequalities for convex and log-convex functions, Linear Algebra Appl., 424, (2007), 25-35.

[2] R. Bhatia, Matrix Analysis, Springer-Verlag, New York (NY), 1997.

[3] R. Bhatia And F. Kittaneh, The singular values of $A+B$ and $A+i B$, Linear Algebra Appl., 431, (2009), 1502-1508.

[4] J. C. Bourin AND E. RiCARD, An asymmetric Kadison's inequality, Linear Algebra Appl., 433, (2010), 499-510.

[5] J. C. BOURIN AND E.-Y. LEE, Unitary orbits of Hermitian operators with convex and concave functions, Bull. London Math. Soc., 44, (2012), 1085-1102.

[6] S. DRURY AND M. Lin, Singular value inequalities for matrices with numerical ranges in a sector, Oper. Matrices, 8, (2014), 1143-1148.

[7] H. X. FU AND Y. LiU, Rotfel'd inequality for partitioned matrices with numerical ranges in a sector, Linear Multilinear Algebra, 64, (2016), 105-109.

[8] A. George And Kh. D. Ikramov, On the properties of Accretive-Dissipative Matrices, Math. Notes, 77, (2005), 767-776.

[9] KH. D. IKramov, Determinantal Inequalities for Accretive-Dissipative Matrices, J. Math. Sci., 121, (2004), 2458-2464.

[10] KH. D. IKRAMOV AND V. N. Chugunov, Inequalities of Fisher and Hadamard types for accretivedissipative matrices, Dokl. Ross. Akad. Nauk, 384, (2002), 585-586.

[11] E.-Y. LeE, Extension of Rotfel'd theorem, Linear Algebra Appl., 435, (2011), 735-741.

[12] C. K. Li AND N. S. SzE, Determinantal and eigenvalue inequalities for matrices with numerical ranges in a sector, J. Math. Anal. Appl., 410, (2014), 487-491.

[13] M. Lin, Reversed determinantal inequalities for accretive-dissipative matrices, Math. Inequal. Appl., 15, (2012), 955-958.

[14] M. LiN, Fischer type determinantal inequalities for accretive-dissipative matrices, Linear Algebra Appl., 438, (2013), 2808-2812.

[15] M. Lin, Extension of a result of Haynsworth and Hartfiel, Arch. Math., 104, (2015), 93-100.

[16] M. LiN AND D. ZHOU, Norm inequalities for accretive-dissipative operator matrices, J. Math. Anal. Appl., 407, (2013), 436-442.

[17] R. C. Thompson, Convex and concave functions of singular values of matrix sums, Pacific J. Math., 1, (1976), 285-290.

[18] F. ZHANG, A matrix decomposition and its application, Linear Multilinear Algebra 63, (2015), 20332042. 
[19] P. ZHANG, A further extension of Rotfel'd theorem, Linear Multilinear Algebra, 63, (2015), 25112517.

[20] P. ZHANG, Extension of Matic's results, Linear Algebra Appl., 486, (2015), 328-334. 